\title{
Does Making Method of Alginate Hydrogel Influence the Chondrogenic Differentiation of Human Mesenchymal Stem Cells?
}

\author{
Jessica Schiavi ${ }^{1}$, Naceur Charif ${ }^{2}$, Natalia de Isla ${ }^{2}$, Danièle Bensoussan ${ }^{2}$, Jean-François Stoltz ${ }^{2}$, \\ Nadia Benkirane-Jessel ${ }^{1}$, Céline Huselstein ${ }^{2}$ \\ ${ }^{1}$ INSERM-UMR, Faculty of medicine, Strasbourg University, Strasbourg, France \\ ${ }^{2}$ Université de Lorraine, CNRS-UMR- Campus Santé - Biopôle, Faculty of medicine, Lorraine University, Vandœuvre-lès-Nancy, France \\ Email: Celine.Huselstein@univ-lorraine.fr
}

Received 2012

\begin{abstract}
To overcome cartilage injury, strategies have been developed in the last few years based on tissue engineering to rebuild the defects. Cartilage engineering is principally based on three main biological factors: cells (native cells (chondrocytes) or a more primitive ones as mesenchymal stem cells), scaffolds and functionalization factors (growth factors, mechanical stimulation and/or hypoxia). Cartilage tissue engineering strategies generally result in homogeneous tissue structures with little resemblance to native zonal organization of articular cartilage. The main objective of our work concerns the buildup of complex biomaterials aimed at reconstructing biological tissue with three dimensional cells construction for mimicking cartilage architecture. Our strategy is based on structures formation by simple and progressive spraying of mixed alginate hydrogel and human mesenchymal stem cells (hMSC). In this work, the comportment of cells and more precisely their chondrogenic differentiation potential is compared to a traditional making process: the mold. We report here that spraying method allowed to product a scaffold with hMSC that confer a favorable environment for neocartilage construction.
\end{abstract}

Keywords: Component: Cartilage Tissue Engineering; Sodium Alginate; Sprayed method; Molded Method

\section{Introduction}

Healthy cartilage is a complex tissue with well-defined functions allowing principally harmonious movements of the articulations over the whole life. Acute or repetitive blunt trauma and excessive impact loading can cause irreversible alterations in articular cartilage matrix. The lack of blood vessels and the inability of chondrocytes (cartilage cells) to repair significantly tissue defects limit the response of cartilage injury. Tissue engineering aims at repairing or replacing damaged or diseased tissue which present a limited self-repair capacity like cartilage. Cells, scaffold materials or both form the basis of this approach, [tritz1, 2]. For cartilage tissue engineering, the use of mature cell types such as chondrocytes is associated with several drawbacks. The limited availability, donor site morbidity, dedifferentiation and limited proliferative capacity urged researchers to study other cell types, particularly mesenchymal stem cells, [3]. Hydrogels provide advantages as their capacity of shaping, supporting chondrocytic phenotype, transducing mechanical loads to cells and their biodegradability, [4, 5]. Alginate hydrogel can be composed with different proteins, GAG (hyaluronic acid), natural or synthetic polymer to mimic tissue composition, have specific mechanical properties or inducing differentiation, [6]. Whereas, most studies using these scaffolds obtain homogenous structure without zonal variation as cartilage in vivo, $[7,8]$. Recently, investigations have considered cartilage organization in order to create stratified tissue engineering which attempts a layered design, [5, 9-13]. How- ever, up to now, few methodologies efficiently synthesizing stratified structures including viable specific cells interacting with a biofunctionalized environment has been proposed. An easily applicable technique that is able to deposit multiple cell types in 3-D without overt cellular damage is probably cell spraying [14].

The goal of the present work is based on structures formation by simple and progressive spraying of mixed alginate hydrogel and human mesenchymal stem cells (hMSC) and to compare their chondrogenic differentiation potential to a traditional making process: the mold. The differentiation potential of human mesenchymal stem cells was characterized particularly for their interest for cartilage engineering.

\section{Materials and Methods}

\subsection{Extraction and Culture of Mesenchymal Stem Cells}

Mesenchymal Stem Cells from human donors were isolated from bone marrow obtained from total hip or knee replacement surgery. Bone marrow was aspirated and diluted in HBSS. Then, mononuclear cells were counted and the suspension was seeded at 50000 mononuclear cells/ $\mathrm{cm}^{2}$ with complete medium (Dulbecco's Modified Eagles Medium low glucose supplemented with $10 \%$ Fetal Bovine Serum (Gibco, France), Penicillin at $100 \mathrm{U} / \mathrm{mL} /$ Streptomycin at $100 \mu \mathrm{g} / \mathrm{mL}(\mathrm{P} / \mathrm{S})$, Amphotericin B (Amp) at $2.5 \mu \mathrm{g} / \mathrm{mL}, 2 \mathrm{mM}$ of Glutamine, and $1 \mathrm{ng} / \mathrm{mL}$ of FGF). hMSC were cultivated up to confluence, passage 0 (P0), 
and detached with trypsin and seeded with complete medium at 1000 cells $/ \mathrm{cm}^{2}, \mathrm{P} 1$. Cells expansion was done up to P3, then cells were detached from the support and embedded in Alginate/Hyaluronic Acid hydrogel (Alg/HA).

\subsection{Construction of Scaffolds Seeded with Cells}

Scaffolds were build-up with 1.5\% Alginate/Hyaluronic Acid (ratio 4:1, Alg/HA). Whatever the making method (spray or mold), hMSC were seeded at $3 \times 10^{6}$ cells/mL of Alg/HA hydrogel.

Spraying construction: The sraying method was previously used and described with other cells type, $[15,16]$. In brief, cells suspension was sprayed at 0.9 bar on a sterile glass plate with an airbrush connected to a compressor. After the glass plate deeping in a $\mathrm{CaCl}_{2}$ bath at $102 \mathrm{mM}$, the gelation was done during $15 \mathrm{~min}$, then the hydrogel was washed twice with a $0.9 \%$ $\mathrm{NaCl}$ solution supplemented with $10 \mathrm{mM}$ Tris at $\mathrm{pH}$ 7.4.

Molding construction: The first type of construction was composed of alginate gel injected into a mold. This approach is often used in cartilage bioengineering. [17,18] 1.5-2 mL of sterile $1.5 \%$ sodium alginate solution are injected into molds (thickness $=1.5 \mathrm{~mm}$, diameter $=25 \mathrm{~mm}$; Bern, Switzerland). [17] Then, alginate suspension gelled immediately on contact with the $\mathrm{CaCl}_{2}$ solution (102 mM), during $15 \mathrm{~min}$.

Whatever scaffolds, cylinders were cut at $5 \mathrm{~mm}$ diameter with a biopsy punch (Stiefel), and were cultivated up to use with differentiation medium (DMEM high glucose, antibiotic, 2 $\mathrm{mM}$ of L-glutamine, $100 \mu \mathrm{g} / \mathrm{mL}$ of sodium pyruvate, $40 \mu \mathrm{g} / \mathrm{mL}$ of L-Proline, $50 \mu \mathrm{g} / \mathrm{mL}$ of L-acid ascorbic, $100 \mathrm{nM}$ of dexamethasone and $1 \mathrm{mM}$ of $\mathrm{CaCl}_{2}$ ), changed twice in a week.

\subsection{Analyze of Cells Viability and Immunophénotyping by Flow Cytometry}

Apoptosis and necrosis of cells were analyzed by flow cytometry using the Vybrant/Apoptosis ${ }^{\mathrm{TM}}$ kit based on Annexin V/Propidium Iodide staining procedure (Molecular Probes, France).

To determine the typical cell surface epitope profile after monolayer expansion and after 3 days of culture in sprayed Alg/HA hydrogels, standard flow cytometer techniques (FACSCalibur; BD biosciences, France) were used.

\subsection{Metabolic Activity}

Mitochondrial activities were measured with Alamar Blue assay (AbD Serotec Ltd, UK) on sample (one cylinder of $5 \mathrm{~mm}$ diameter) during the 28 days of culture. After $4 \mathrm{~h}$ of scaffolds incubation with Alamar Blue solution $(10 \% \mathrm{v} / \mathrm{v})$ at $37^{\circ} \mathrm{C}$, the solution was subsequently removed and Alamar Blue absorbance is read at wavelength $570 \mathrm{~nm}$ (reduced compound absorbance) and $600 \mathrm{~nm}$ (oxidized compound absorbance) with a spectrophotometer (Varioskan Flash, Thermo Scientific). Results were standardized with the quantity of DNA present by sample.

\subsection{Extracellular Matrix Synthesis}

Cells were extracted from hydrogel by chelating $\mathrm{Ca}^{2+}$ in alginate eggbox bindings and were washed with PBS 3 fold. First,
RNA extraction was done with the RNeasy extraction kit (Qiagen), and purified with a DNase kit (Qiagen). Then, cDNA were obtained thanks to the reverse-transcription of mRNA with the iscript kit (Biorad). Finally, a qRT PCR was done with the Quantitec SYBR Green PCR kit (Qiagen) and cDNA obtained from samples, on a Light Cycler system (Roche Diagnostics) during 45 cycles to analyze quantitatively gene expression. All gene expression was reported to the housekeeping gene RP-29.

\subsection{Statistical Analysis}

All data are presented as means \pm standard error means (SEM) of at least three independent experiments. A second way ANOVA was used to determine if significant differences existed for the mixed-population experiment and a Bonferroni posttest was performed to evaluate significance for all experiments.

\section{Results}

To examine surface molecule expression on hMSC, flow cytometric analysis was used to show the proportion of cells positive for hMSC markers (CD166, CD105, CD44 and CD73) at the end of monolayer culture (P3) and after 3 days of culture in sprayed Alg/HA hydrogels (Figure 1). It appeared that the proportion of positive cells for CD105 significantly decreased when cells were in a $3 \mathrm{D}$ matrix compared to the monolayer culture. On the other hand, the proportion of cells expressing CD73, CD44 and CD166 markers increased significantly in sprayed Alg/HA hydrogel compared to the monolayer culture. Whatever the kinds of culture, hMSC were negative for leukocyte marker CD45, hematopoietic stem cell markers CD34 thus verifying the lack of contaminating hematopoietic cells. Cells were also negative for HLA-DR.
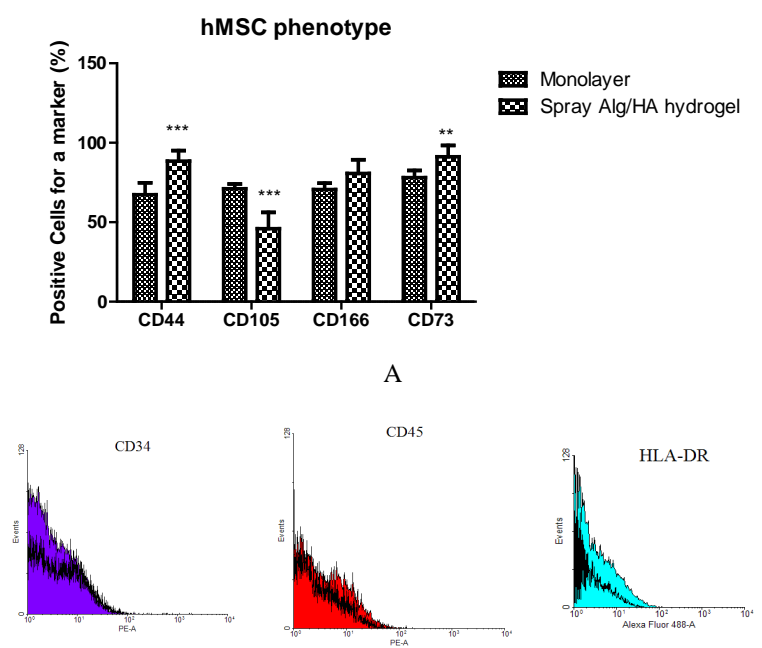

B

Figure 1. Flow cytometry analysis for the expression of cell surface markers related to mesenchymal stem cells (CD44, CD73, CD105 and CD166; Fig. A), hematopoietic stem cells (CD34, Fig. B), leukocytes (CD45, Fig. B), and HLA-DR (Fig. B) on hMSC at the end of monolayer culture (P3) and in sprayed Alg/HA hydrogels (on day 3). Data are presented as mean \pm SEM. 
hMSC viability in sprayed Alg/HA hydrogels was followed during 28 days of culture, and was compared to cell viability in molded Alg/HA hydrogels (Figure 2). After 3 days of culture, whatever the scaffold, cell viability was always higher than $70 \%$. Alamar Blue test was done to measure mitochondrial activity of samples and metabolic activity was obtained by taking account the DNA content. During the first week of culture, metabolic activity was homogenous between each scaffold and still weak. After 14 days of culture, hMSC embedded in the sprayed hydrogels were more active than hMSC embedded in the molded ones. Then, metabolic activity increased up to the end of the experiment in sprayed hydrogels, with an increase 2.8 times higher than the molded hydrogels.

Quantitative RT-PCR was performed for gene expression analysis on sprayed and molded Alg/HA hydrogels after 3 and 14 days of culture. The mean relative gene expression of aggrecan, collagen I, collagen II, Cartilage Oligomeric Matrix Protein (COMP), Sox9, in the scaffolds is presented in Figure 3.

After 3 days of culture, any difference was observed for the mean mRNA expression of aggrecan, collagen I, COMP, Sox9 in Alg/HA hydrogels, whatever the making method. In contrast, after 14 days of culture, the mRNA expression levels of aggrecan, COMP, collagen I and Sox9 were higher in hMSC embedded in sprayed hydrogels than in molded ones.

\section{Discussion}

Alginate hydrogel is a polymer largely employed for it is very easy to use in cartilage engineering. $[19,20]$ However, few investigations considered the organization of cartilage as a basis for the development of the stratified tissue engineering. From this viewpoint, complex 3-D structures of cartilage can a priori be built layer by layer using a large number of different components, including hydrogel with various cell types, drugs, proteins, peptides or DNA. However, few methodologies efficiently synthesizing stratified structures including viable specific cells interacting with a biofunctionalized environment has been proposed. An easily applicable technique that is able to deposit multiple cell types in 3-D without over cellular damage is probably cell spraying. Recently, we have reported the spray formation of complex 3D multilayers, composed of alternating calcium-alginate gel domains containing cells $[14,16]$.

In this study, we compared the effect of sprayed method on
hMSC behavior (phenotype, viability, metabolic activity) and differentiation potential in chondrogenic lineage (Sox9 and extracellular matrix markers expressions) to a more traditional making process: the mold [17]. hMSC are largely used in tissue engineering in particular in cartilage engineering. Indeed, native cartilage cells, chondrocytes, are a limited source and can induce fibrocartilage in the taking area, thus we use hMSC for the easier ability to obtain a large number of cells, as other research ways, [7,8]. Our data showed that, after 3 days of culture in sprayed Alg/HA hydrogels, hMSC obtained expressed a panel of conventionally used mesenchymal stem cell surface markers. Moreover, after 3 days of culture, it seems that cells were adapted to their environment without any detectable damage. This hypothesis could be confirmed by cell metabolism results. Indeed, we observed, from D14 to D28, significant increase of cell metabolism when cells were embedded in sprayed hydrogel. This metabolism is higher than in molded hydrogels. In addition, the differentiation of the hMSC in chondrocyte seems to begin because relative expression of cartilage genes (Sox9, COMP, Aggrecan, Collagen II) increases significantly up to 14 days in sprayed Alg/HA hydrogels. In a previous study, we showed that making method influence the scaffolds mechanical behavior. In fact, the mechanical performances of sprayed hydrogels was enhanced compared to molded ones [14]. It is well-known that cell mechanical environment is important for various cell activities, such as survival, migration, signal transduction but also cell differentiation. This new making method allowed having a tridimensional structure and a mechanical environment in favor of chondrogenic differentiation.
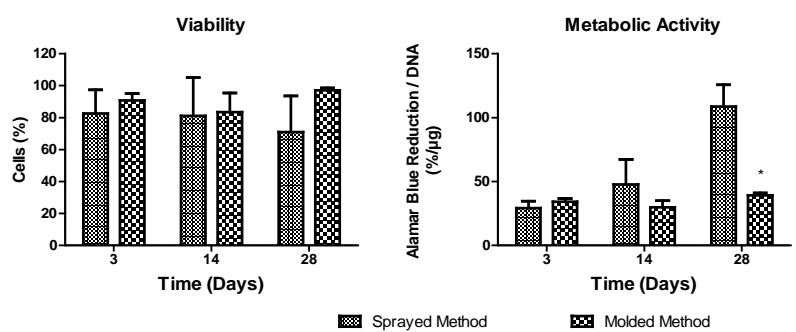

Figure 2. hMSCs viability and metabolism during 14 days of culture in alginate scaffolds obtained either by sprayed or molded method. Data are presented as mean \pm SEM.

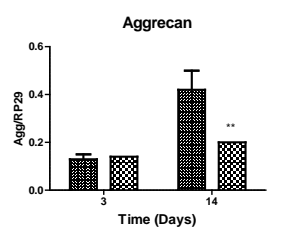

Sox 9
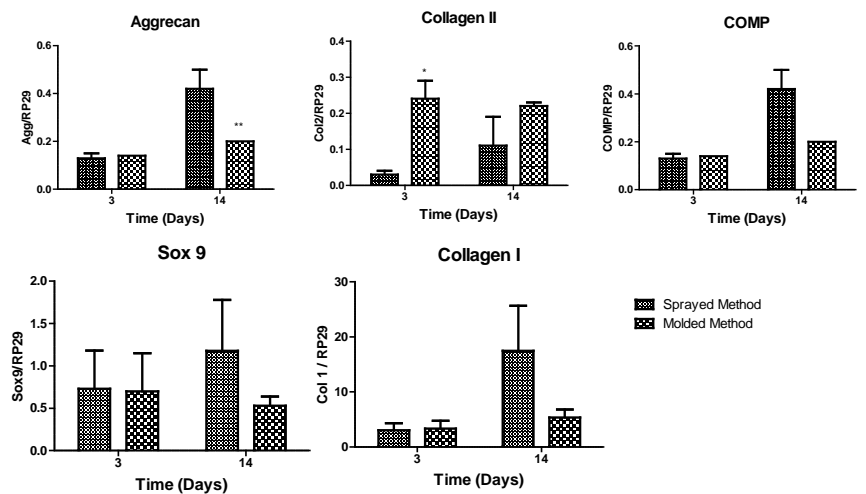

Collagen I
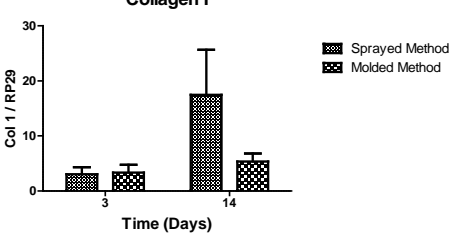

Sprayed Method

Figure 3. Real-time PCR analysis of cDNA derived from hMSC embedded in Alg/HA hydrogels. 3D hydrogels were performed with sprayed or molded method and maintened 14 days in culture. Data are presented as mean \pm SEM. 
The proposed way will allow to control from the surface to the in-depth of the distribution of the different needed elements (matrix and cells). This strategy is important for chondrogenesis induction of hMSC without growth factor, [8], it will permit also building up a scaffold and gives configuration to mimic cartilage structure.

\section{Acknowledgements}

This work was supported by the "Lorraine region" grant. N. Jessel is indebted to CHU de Nancy, Hôpital Central, orthopedic surgery (Contrat d'interface INSERM-CHU).

\section{REFERENCES}

[1] C. Chung and J. A. Burdick, "Engineering cartilage tissue," Adv Drug Deliv Rev, vol. 60, pp. 243-62, 2008.

[2] R. Langer and J. P. Vacanti, "Tissue engineering," Science, vol. 260, pp. 920-6, 1993

[3] L. D. Solorio, A. S. Fu, R. Hernández-Irizarry, and E. Alsberg, "Chondrogenic differentiation of human mesenchymal stem cell aggregates via controlled release of TGF-beta1 from incorporated polymer microspheres," Journal of Biomedical Materials Research Part A, vol. 92A, pp. 1139-1144, 2009.

[4] E. Fragonas, M. Valente, M. Pozzi-Mucelli, R. Toffanin, R. Rizzo, F. Silvestri, and F. Vittur, "Articular cartilage repair in rabbits by using suspensions of allogenic chondrocytes in alginate," Biomaterials, vol. 21, pp. 795-801, 2000.

[5] [5] E. Han, W. C. Bae, N. D. Hsieh-Bonassera, V. W. Wong, B. L. Schumacher, S. Gortz, K. Masuda, W. D. Bugbee, and R. L. Sah, "Shaped, stratified, scaffold-free grafts for articular cartilage defects," Clin Orthop Relat Res, vol. 466, pp. 1912-20, 2008.

[6] N. S. Hwang, S. Varghese, H. J. Lee, P. Theprungsirikul, A. Canver, B. Sharma, and J. Elisseeff, "Response of zonal chondrocytes to extracellular matrix-hydrogels," FEBS Lett, vol. 581, pp. 4172-8, 2007.

[7] K. S. Stok, G. Lisignoli, S. Cristino, A. Facchini, and R. Muller, "Mechano-functional assessment of human mesenchymal stem cells grown in three-dimensional hyaluronan-based scaffolds for cartilage tissue engineering," J Biomed Mater Res A, vol. 93, pp. 37-45, Apr 2009.

[8] A. Derfoul, G. L. Perkins, D. J. Hall, and R. S. Tuan, "Glucocorticoids promote chondrogenic differentiation of adult human mesenchymal stem cells by enhancing expression of cartilage extracellular matrix genes," Stem Cells, vol. 24, pp. 1487-95, Jun 2006.

[9] C. S. Lee, J. P. Gleghorn, N. Won Choi, M. Cabodi, A. D. Stroock, and L. J. Bonassar, "Integration of layered chondrocyte-seeded alginate hydrogel scaffolds," Biomaterials, vol. 28, pp. 2987-93, 2007.
[10] K. W. Ng, C. C. B. Wang, R. L. Mauck, T.-A. N. Kelly, N. O. Chahine, K. D. Costa, G. A. Ateshian, and C. T. Hung, "A layered agarose approach to fabricate depth-dependent inhomogeneity in chondrocyte-seeded constructs," Journal of Orthopaedic Research, vol. 23, pp. 134-141, 2005.

[11] B. A. Harley, A. K. Lynn, Z. Wissner-Gross, W. Bonfield, I. V. Yannas, and L. J. Gibson, "Design of a multiphase osteochondral scaffold III: Fabrication of layered scaffolds with continuous interfaces," J Biomed Mater Res A, vol. 92, pp. 1078-93, Mar 1.

[12] T. J. Klein, S. C. Rizzi, J. C. Reichert, N. Georgi, J. Malda, W. Schuurman, R. W. Crawford, and D. W. Hutmacher, "Strategies for zonal cartilage repair using hydrogels," Macromol Biosci, vol. 9, pp. 1049-58, Nov 102009.

[13] T. J. Klein, B. L. Schumacher, T. A. Schmidt, K. W. Li, M. S. Voegtline, K. Masuda, E. J. Thonar, and R. L. Sah, "Tissue engineering of stratified articular cartilage from chondrocyte subpopulations," Osteoarthritis Cartilage, vol. 11, pp. 595-602, Aug 2003.

[14] J. Tritz, R. Rahouadj, N. d. Isla, N. Charif, A. Pinzano, D. Mainard, D. Bensoussan, P. Netter, J.-F. Stoltz, N. Benkirane-Jessel, and C. Huselstein., "Designing a three-dimensional alginate hydrogel by spraying method for cartilage tissue engineering," Soft Matter, vol. 6, pp. 5165-5174, 2010.

[15] H. Mjahed, C. Porcel, B. Senger, A. Chassepot, P. Netter, P. Gillet, G. Decher, J.-C. Voegel, P. Schaaf, N. Benkirane-Jessel, and F. Boulmedais, "Micro-stratified architectures based on successive stacking of alginate gel layers and poly(L-lysine)-hyaluronic acid multilayer films aimed at tissue engineering," Soft matter, vol. 4, pp. 1422-1429, 2008.

[16] J. Tritz-Schiavi, N. Charif, C. Henrionnet, N. de Isla, D. Bensoussan, J. Magdalou, N. Benkirane-Jessel, J. F. Stoltz, and C. Huselstein, "Original approach for cartilage tissue engineering with mesenchymal stem cells," Biomed Mater Eng, vol. 20, pp. 167-74, 2010.

[17] M. Wong, M. Siegrist, X. Wang, and E. Hunziker, "Development of mechanically stable alginate/chondrocyte constructs: effects of guluronic acid content and matrix synthesis," J Orthop Res, vol. 19, pp. 493-9, 2001.

[18] S. C. N. Chang, J. A. Rowley, G. Tobias, N. G. Genes, A. K. Roy, D. J. Mooney, C. A. Vacanti, and L. J. Bonassar, "Injection molding of chondrocyte/alginate constructs in the shape of facial implants," Journal of Biomedical Materials Research, vol. 55, pp. 503-511, 2001.

[19] G. M. Williams, T. J. Klein, and R. L. Sah, "Cell density alters matrix accumulation in two distinct fractions and the mechanical integrity of alginate-chondrocyte constructs," Acta Biomater, vol. 1, pp. 625-33, 2005.

[20] Y. Wang, N. de Isla, C. Huselstein, B. Wang, P. Netter, J. F. Stoltz, and S. Muller, "Effect of alginate culture and mechanical stimulation on cartilaginous matrix synthesis of rat dedifferentiated chondrocytes," Biomed Mater Eng, vol. 18, pp. S47-54, 2008. 